\title{
TUMEUR MALIGNE DES GAINES NERVEUSES PÉRIPHÉRIQUES RÉVÉLANT UNE NEUROFIBROMATOSE TYPE 1
}

\author{
I. CHARFEDDINE, M. MNEJJA, B. HAMMAMI, AM. CHAKROUN, S. KALLEL, A. HADJ KACEM*, \\ I. FRIKHA ${ }^{\star}$, S. MAKNI ${ }^{\star *}$, T. BOUDAWARA**, A. CHAKROUN, A. GHORBEL. \\ SERVICE D'ORL ET CHIRURGIE MAXILLO-FACIALE \\ *SERVICE DE CHIRURGIE CARDIOVASCULAIRE \\ **LABORATOIRE D'ANATOMIE ET CYTOLOGIE PATHOLOGIQUES \\ CHU HABIB BOURGUIBA, SFAX, TUNISIE.
}

\begin{abstract}
La Neurofibromatose type 1 (NF1) ou maladie de Von Recklinghausen est une affection à expression clinique variable. La transformation maligne est rare et redoutable. Le but de ce travail est d'étudier les critères diagnostiques ainsi que la prise en charge thérapeutique de cette transformation maligne.

Observation : A.B, âgé de 16 ans, a consulté pour une tuméfaction basicervicale antérieure d'apparition récente, ayant augmenté progressivement de taille sans signes de compression ni de dysthyroïdie.

L'examen clinique a trouvé une masse dure, bien limitée, indolore, de $4 \mathrm{~cm}$ de grand axe siégeant en regard du manubrium sternal, adhérente au plan profond et débordant sur le creux sus sternal. A cette masse s'associaient des taches cutanées café au lait disséminées sur tout le corps, des nodules de Lish iriens et des neurofibromes occipitaux et parotidiens.

Après une exérèse de la masse par voie cervicale, l'examen histologique a conclu à une tumeur maligne de la gaine nerveuse. Le malade a eu dans un 2ème temps, une exérèse plus large emportant le manubrium sternal et les tissus adjacents avec une reconstruction par des fragments de côtes mais avec des limites chirurgicales envahies. L'évolution a été marquée par une poursuite évolutive précoce imposant une exérèse chirurgicale plus large suivie d'une chimiothérapie et d'une radiothérapie externe (RT). Le malade était cependant toujours en poursuite évolutive et le décès est survenu dans un contexte d'envahissement médiastinal.
\end{abstract}

Mots-clés : Neurofibromatose type 1, Sarcome, tissus mous, gaines nerveuses périphériques, Chirurgie.

\begin{abstract}
Neurofibromatosis type 1 or Von Recklinghausen's disease is an affection with variable clinic expression. Malignant transformation is rare and dangerous. The aim of this work is to study diagnostic criteria as well as treatment of this malignant transformation.

Case report : A sixteen years old patient showed with gradually progressive basicervical mass without compression nor dysthyroidy signs. Physical examination revealed a painless, hard and well limited mass measuring $4 \mathrm{~cm}$. It's located in front of sternal manubrium and associated with multiples "café au lait" spots, iris Lisch nodules and generalized neurofibromas.

This mass was excised by cervical surgery. The histological exam revealed a malignant peripheral nerve sheath tumor. A larger resection was performed carrying away the sternal manubrium and adjacent tissues with a reconstruction by bits of ribs but within invaded surgical limits. Precocious recurence has observed 3 months later which was treated by surgery, chemotherapy and radiotherapy but the patient's tumor was still progressing. He died of mediastinal invasion two months later.
\end{abstract}

Keywords : Neurofibromatosis type 1, sarcoma, Soft tissue, Peripheral nerve sheath, Surgery.

\section{INTRODUCTION}

La neurofibromatose 1 (NF1) ou maladie de Von Recklinghausen est une affection autosomique dominante caractérisée par une extrême variabilité de son expression clinique. Le tableau clinique associe, le plus souvent de multiples taches cutanée café au lait, des lentigines axillaires et inguinales, des neurofibromes cutanés et des nodules iriens de Lisch.

Les tumeurs malignes des gaines nerveuses périphériques (TMGNP), anciennement appelées neurofibrosar- comes, constituent la principale complication de la NF1 à l'âge adulte. Leur incidence chez les malades atteints de NF1 est de 5 à $10 \%(1,2,3,4,5,6)$.

Les autres TMGNP sont radio induites ou solitaires, appelées encore "de novo".

Nous rapportons, dans cet article une observation de NF1 révélée par une TMGNP. 


\section{OBSERVATIONS}

Un enfant âgé de 6 ans, sans antécédents pathologiques notables et notamment sans notion de traumatisme ni d'irradiation, a été hospitalisé en Mai 1997 pour des céphalées intenses et une diminution de l'acuité visuelle. L'examen cutané a révélé des tâches café au lait diffuses. L'examen ophtalmologique a montré une diminution de l'acuité visuelle avec un fond d'œil normal. L'échographie cervicale était normale et l'IRM cérébrale a mis en évidence des lésions bilatérales nodulaires en hypersignal T2 des noyaux lenticulaires. II n'y avait pas de lésions des angles pontocérébelleux ni des voies optiques.

Le diagnostic de neurofibromatose type 1 a été évoqué mais non retenu.

En juillet 2006, il a reconsulté pour une tuméfaction douloureuse du creux sus-sternal évoluant depuis 2 mois. L'examen a trouvé une masse dure bien limitée adhérente au sternum faisant $4 \mathrm{~cm}$ de grand axe. II n'y avait pas de déficit neurologique. A cette masse s'associaient en plus des multiples tâches café au lait disséminées sur tout le corps, des nodules de Lish iriens et des neurofibromes occipitaux et parotidiens caractéristiques de la neurofibromatose type 1.

Le scanner a montré une masse de densité tissulaire, homogène et bien limitée au contact de la fourchette sternale se réhaussant intensément après injection de produit de contraste sans lyse osseuse en regard (fig.1).

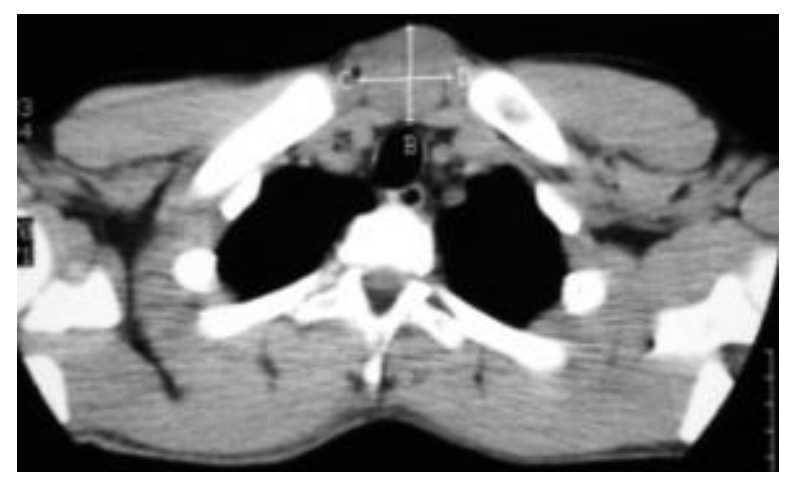

Fig.1 : TDM en coupe axiale sans injection de produit de contraste : masse de densité tissulaire homogène et bien limitée sans lyse osseuse.

Le malade a eu une résection de la masse par voie cervicale. La dissection était facile mais la masse paraissait adhérente aux muscles présternaux.

L'examen anatomopathologique de la pièce opératoire a conclu à une tumeur maligne des gaines nerveuses périphériques de grade I selon la fédération nationale des centres de lutte contre le cancer (FNCLCC), développée sur un neurofibrome plexiforme avec envahissement des tissus fibro-adipeux et musculaires. Les limites chirurgicales étaient envahies. Nous avons réopéré le malade réalisant une manubriectomie sternale avec reconstruc- tion par des segments de côtes et plastie de recouvrement par le muscle grand pectoral droit (fig. 2).

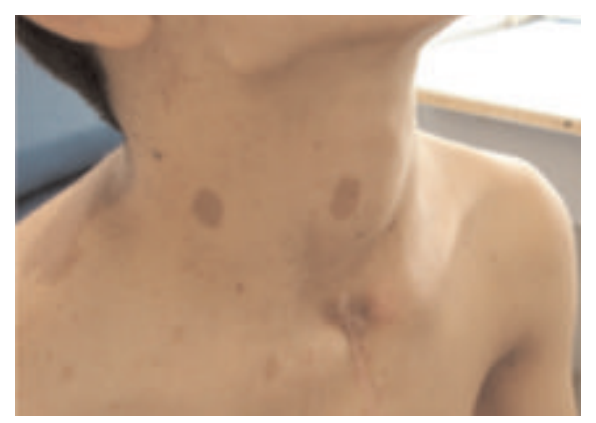

Fig.2 : Vue post-opératoire : Cicatrice cervico-thoracique associée à des multiples tâches cutanées café au lait.

Les limites chirurgicales au niveau du tissu musculaire cervical étaient encore tumorales. Nous avons décidé de ne pas réopérer le malade, sous réserve d'une surveillance rigoureuse clinique et radiologique. Une radiothérapie adjuvante n'a pas été réalisée afin d'éviter un éventuel passage d'un bas grade à un haut grade. Mais l'évolution a été marquée par une poursuite évolutive précoce. En effet, après un délai de 3 mois, le malade a présenté une tuméfaction susclaviculaire gauche de $4 \mathrm{~cm}$ de diamètre, dure, fixée au plan profond et une autre tuméfaction de $7 \mathrm{~cm}$ en regard de la plastie sternale et de mêmes caractéristiques (fig.3,4).

Une exérèse plus large a été réalisée emportant le manubrium sternal et les tissus adjacents avec une reconstruction par des fragments de côtes et plastie de recouvrement par le muscle grand pectoral. Les limites chirurgicales étaient toujours tumorales.

Nous avons complété par trois cures de chimiothérapie au cours desquelles le malade a présenté une énorme progression tumorale. A la fin de la 3ème cure, le malade présentait une lésion polylobée en perméation cutanée faisant 22x22cm envahissant la paroi thoracique (fig.5). Une RT externe palliative à la dose de 30 Gy en 10 séances a été réalisée sans effet sur la progression tumorale. Deux mois après, le malade est décédé dans un tableau d'arrêt cardio-respiratoire par envahissement médiastinal.

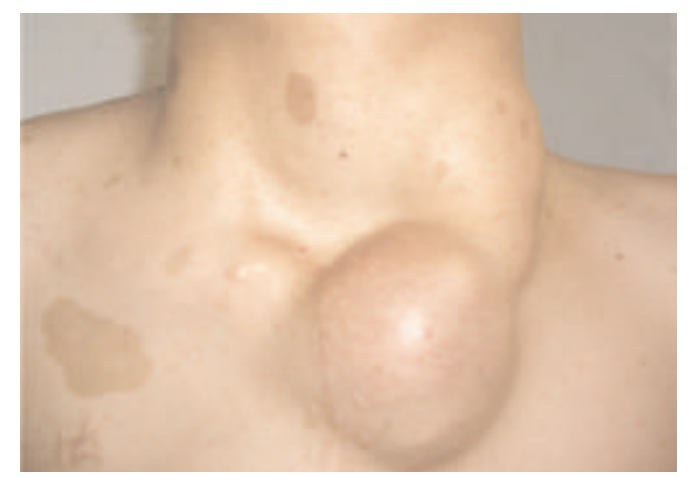

Fig.3 : Poursuite évolutive locale 3 mois après chirurgie. 


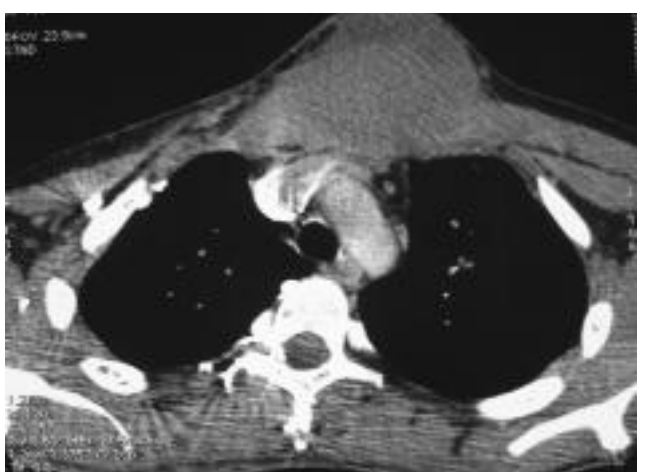

Fig.4 : TDM thoracique en coupe axiale et après injection de produit de contraste : poursuite évolutive 3 mois après la chirurgie.

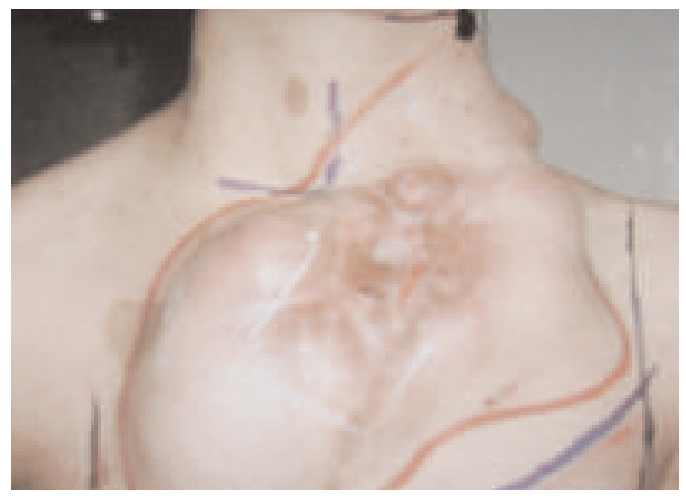

Fig.5 : Aspet après chimiothérapie.

\section{DISCUSSION}

L'incidence des tumeurs malignes des gaines nerveuses périphériques (TMGNP) est de 5 à $10 \%$ chez les patients ayant une NF1 alors qu'elle est de 0,001\% dans la population générale $(1,2,3,4,5)$. Elles représentent moins de $5 \%$ de tous de les sarcomes des tissus mous (7). II s'agit typiquement de tumeurs de l'adulte jeune, âgé de 20 à 50 ans $(1,8)$. Des cas ont été rapportés chez l'enfant et chez les personnes âgées (8). II n'y a pas de prédominance de sexe.

Trente à $40 \%$ des TMGNP résultent de la transformation d'un neurofibrome plexiforme en particulier dans sa forme géante, multiple et extensive, ceci impose d'ailleurs une surveillance stricte et prolongée des malades ayant une NF1. Les neurofibromes plexiformes sont présents dans 30 à $50 \%$ des NF1 et peuvent dégénérer en tumeur maligne des gaines nerveuses dans 10 à $15 \%(9,10)$. Ce risque de transformation maligne est néanmoins moins important pour les neurofibromes nodulaires.

L'évolution naturelle d'un neurofibrome plexiforme est divisée en deux phases : une phase dite de quiescence et une phase active pendant laquelle le neurofibrome augmente rapidement de taille sans être toujours un prélude à la malignité.

II est actuellement parfaitement établi que c'est le gène NF1 (gène suppresseur de tumeur), localisé sur le bras long du chromosome 17 et codant pour la protéine "neurofibromine" qui intervient dans la genèse des TMGNP $(7,11,12)$.

Les signes d'appel d'une transformation maligne sont une augmentation rapide de taille d'un neurofibrome ancien ou nouvellement apparu, une induration, des douleurs, l'apparition ou la modification des signes neurologiques préexistants (13). Toutefois ces tumeurs périphériques peuvent être asymptomatiques.

Concernant la localisation de ces TMGNP, elles se répartissent également entre les membres et le tronc (25\%). La confirmation de diagnostic est apportée par l'examen histologique. Macroscopiquement, les TMGNP se présentent sous forme d'une masse fusiforme ou ovoïde englobant un nerf. Elles sont composées de cellules arrangées en faisceaux associées à des foyers de nécrose, d'hémorragie et de calcification. Les critères de malignité incluent l'invasion des structures de voisinage, les emboles vasculaires, le pléomorphisme nucléaire, la nécrose et la présence de mitoses. Le système de grading préconisé par la FNCLCC repose sur trois paramètres : la différenciation cellulaire, la nécrose et le nombre de mitoses (14). Ce système de grading reste le meilleur prédicteur de la survie. Les analyses immunohistochimiques montrent une réactivité à la protéine S-100, à la protéine myéline basique et au Leu-7 positives dans respectivement $58 \%, 47 \%$ et $52 \%$ des TMGNP (1, 8, $15,16,17,18)$.

En ce qui concerne l'imagerie, la TDM et l'IRM précisent la localisation, la taille, les contours de la lésion et ses rapports avec les organes de voisinage, sans permettre cependant d'en affirmer avec certitude la nature maligne ou bénigne. En effet, toutes les tumeurs neurogènes peuvent présenter des calcifications ainsi que des zones de nécrose et d'hémorragie, qui donnent un aspect hétérogène même aux lésions bénignes. D'autre part, les lésions malignes gardent souvent des contours bien limités, ce qui leur donne un aspect faussement rassurant comme c'était le cas pour notre malade. Ces deux examens radiologiques permettent par ailleur de montrer un éventuel envahissement des tissus mous et des structures osseuses de voisinage.

La fluorodéoxyglucose positron émission tomography (FDGPET ), dont le principe repose sur la quantification du glucose dans la cellule tumorale, est une technique non invasive utile dans la surveillance périodique des malades atteints de NF1. Elle permet de détecter une transformation maligne d'un neurofibrome $(1,2)$.

La conduite thérapeutique n'est pas codifiée. Elle inclut la chirurgie et la radiothérapie adjuvante. Tous les auteurs s'accordent sur le fait que le traitement est avant tout chirurgical (9). II est, en effet, important de réaliser une exérèse de toute la lésion en gardant des marges saines afin d'éviter une récidive locale.

La radiothérapie postopératoire est indiquée dans les 
TMGNP de haut grade ou lorsque les limites d'exérèse sont envahies (1). Pour Madhabanda Kar (2), les tumeurs de grande taille ou profondes constituent également une indication à la radiothérapie adjuvante.

La chimiothérapie reste encore un sujet de controverse (20). Elle peut être utile dans le traitement des métastases ou en préopératoire lorsque la tumeur est de grande taille et jugée au début non résécable $(21,22)$.

Dans tous les cas, le pronostic des TMGNP est également mauvais $(23,24)$. Le taux de survie à 5 ans est de 20 à $50 \%$ (15). L'évolution générale dépend du grade histologique de la tumeur, de sa taille, de sa localisation, de l'association ou non à une NF1 et de la possibilité et de la qualité de la chirurgie première (20). Le risque de récidive locale est de 40 à $65 \%$ (22).

Les métastases sont hématogènes ou suivent le trajet des gaines nerveuses. Les métastases à distance sont localisées aux poumons, foie et aux os. Elles apparaissent dans un délai moyen de deux ans, délai d'autant plus court qu'il existe une NF1 $(8,15)$.

\section{REFERENCES}

1. Bilgic B, Ates LE, Demiryont M, Ozger H, Dizdar Y. Malignant peripheral nerve sheath tumors associated with neurofibromatosis type 1. Pathol Oncol Res. 2003, 9(3): 201-5.

2. Kar M, Deo SV, Shukla NK, et al Malignant peripheral nerve sheath tumors (MPNST)--clinicopathological study and treatment outcome of twenty-four cases. World J Surg Oncol. 2006, 22(4): 55.

3. Pinson S, Wolkenstein P. La neurofibromatose 1 (NF1) ou maladie de Von Recklinghausen. La revue de médecine interne. 2005; 26;196-215.

4. Sommelet D. Specificity of benign and malignant tumoral complications of type 1 neurofibromatosis. Arch Pediatr. 2004, 11(6): 550-2.

5 . Tonsgard, JH. Clinical manifestations and management of neurofibromatosis type 1. Semin Pediatr Neurol. 2006, 13(1) : 2-7.

6. Bonnemaison E, Roze-Abert B, Lorette G. Complications de la neurofibromatose de type 1 chez l'enfant : à propos d'une série de 100 cas. Archives de pédiatrie. 2006, (13):1009-1014.

7. Grobmyer SR, Reith JD, Shahlaee A, Bush CH, Hochwald SN. Malignant Peripheral Nerve Sheath Tumor: molecular pathogenesis and current management considerations. J Surg Oncol. 2008, 97(4) : 340-9.

8. Soualhi M, Elouazani H, Chaibainou A, Bouchantouf R. Tumeur maligne des gaines des nerfs périphériques type épithélioide. Rev. Pneumol. Clin. 2004, 60 : 50-54.

9. Minovi A, Basten O, Hunter B, Draf W, Bockmühl U. Malignant peripheral nerve sheath tumors of the head and neck: management of 10 cases and literature review. Head Neck. 2007, 29(5) :439-45.

10. Upadhyaya M, Spurlock G, Monem B, Thomas N, Friedrich RE, Kluwe L, Mautner V. Germline and somatic NF1 gene mutations in plexiform neurofibromas. Hum Mutat. 2008, Vol. 16.

11. Shen MH, Harper PS, Upadhyaya M. Molecular genetics of neurofibromatosis type 1 (NF1). J Med Genet. 1996, 33(1) : 2-17.

12. Zhu Y, Parada LF. Neurofibromin, a tumor suppressor in the nervous system. Exp Cell Res. . 2001, Vol. 264, 1, pp. 19-28.

13. Valeyrie-Allanore L, Ortonne N, Lantieri L, Ferkal S, Wechsler J, Bagot M, Wolkenstein P. Histopathologically dysplastic neurofibromas in neurofibromato- sis 1: diagnostic criteria, prevalence and clinical significance. $\mathrm{Br} \mathrm{J}$ Dermatol. 2008, 158(5) : 1008-12.

14. Collin F, Gelly-Marty M. Sarcomes des tissus mous: données anatomopathologiques actuelles. Cancer/Radiothérapie . 2006, $10: 7-14$

15. Topal O, Yilmaz T, Oğretmenoğlu O. Giant malignant peripheral nerve sheath tumor of the neck in a patient with neurofibromatosis-1. Int $\mathrm{J}$ Pediatr Otorhinolaryngol. 2004, 68(11) : 1465-7.

16. Yamaguchi U, Hasegawa T, Hirose T, Chuman H, Kawai A, Ito Y, Beppu Y. Low grade malignant peripheral nerve sheath tumour: varied cytological and histological patterns. J Clin Pathol. . 2003, 56(11): 826-30.

17. Karpuz V, Letovanec N, Von Hochstetter A, Joris F. Malignant peripheral nerve sheath tumor with rhabdomyoblastic differentiation and glandular component. Ann Pathol. 2000, 20(1) : 62-5.

18. Goujon JM, Bataille B, Menet E, Lapierre F. Neurinomas-neurofibromas. Neurochirurgie. 1997, 43(1) : 35-8.

19. Perrin RG, Guha A. Malignant peripheral nerve sheath tumors. Neurosurg Clin N Am. 2004, 15(2) : 203-16.

20. Pfeiffer J, Arapakis I, Boedeker CC, Ridder GJ. Malignant peripheral nerve sheath tumour of the paranasal sinuses and the anterior skull base. J Craniomaxillofac Surg. 2008, 36(5) : 293-9.

21. Gutmann DH, Hedrick NM, Li J, Nagarajan R, Perry A, Watson MA. Comparative gene expression profile analysis of neurofibromatosis 1-associated and sporadic pilocytic astrocytomas. Cancer Res. . 2002, 62(7) : 2085-91.

22. Malone JP, Lee WJ, Levin RJ. Clinical characteristics and treatment outcome for nonvestibular schwannomas of the head and neck. Am J Otolaryngol. 2005, 26(2):108-12.

23. Beegun I, Bottrill ID, Hollowood K. Malignant peripheral nerve sheath tumours of the infraorbital nerve: case report and literature review. J Laryngol Otol. . 2008, 20:1-5. 24

24. Sabesan T, Hussein K, llankovan V. Malignant peripheral nerve sheath tumour of the parapharyngeal space in a patient with neurofibromatosis type 1. $\mathrm{Br} J$ Oral Maxillofac Surg. 2008;46(7):585-7. 